\section{MUTATION INTIME DE LA PEINTURE DANS LA VIDÉO NUMÉRIQUE Éliane Chiron}

RESUMÉ: Depuis que je réalise des vidéos numériques, suis-je encore peintre? La vidéo fait à chaque fois revenir la peinture. Elle est hantée par des phrases: Les couleurs "font brûler mon cerveau» (Malevitch) et se voient "comme une cicatrice sur un visage " (Cennino Cennini).

Dans une vidéo personnelle intitulée Les Nageuses (20II), comment ont muté la chair et le sang (de l'artiste), matières de l'art de peindre avec tout ce qui choit du corps? Comment tout ce qui dans les ténèbres telluriques, au fond des océans, se métamorphose, magma visqueux qui déferle en plis et fractures gigantesques, s'engouffre et jaillit à chaque seconde, s'accorde-t-il avec la définition de la peinture comme «tache» qui ressort sur le vivant (W. Benjamin)? Comment la description de la piscine par Merleau-Ponty, modèle selon lui de la quête des peintres, s'incarne-t-elle dans cette vidéo? Comment l'intime en art, apparu selon Daniel Arasse dans le tableau de la Renaissance, a-t-il muté dans Les Nageuses? Les références historiques seront nécessaires pour comprendre que peindre en étant contemporain suppose un contact intime avec la matière picturale, fut-elle produite par un code, sans craindre de s'y perdre. Car les codes du numérique sont issus des lois cosmiques et $\mathrm{du}$ cerveau humain.

Nos muqueuses, organes, chromosomes, battements de cœur, viscères? Ce avec quoi en définitive on peint, en utilisant toutes les techniques d'un champ élargi de la peinture, à seule fin que se déploie l'intime mutabilité de la couleur.

MOTS-CLÉS: Contemporain, couleur-cicatrice, intime, lois cosmiques, mutabilité, piscine, tache, vidéo numérique.

"Quand je dis couleur, cela s'entend de toute matière picturale'..

LYOTARD, J. F. L'inhumain. Causeries sur le temps, Paris, Galilée, I988, p. I63.

\section{COULEUR-CICATRICE}

Je n'ai pas appris la peinture à l'école des beaux-arts. Malevitch non plus, ni Picasso. Cézanne échoua au concours d'entrée. Autre époque? Sans doute, mais enfin. Picasso apprit toutes les techniques de l'art (tekhnè) avec son père, directeur de l'école des Arts et Métiers de la Corogne en Espagne; Malevitch préféra comme tous les jeunes artistes de l'époque en Russie, étudier dans une école d'Arts appliqués. On sait qu'il inventa son Carré noir pour la pièce de théâtre Victoire sur le soleil, dans l'effervescence d'échanges un été au bord de la mer Baltique, entre theâtre, poésie, musique et peinture. Lui seul fut capable du transfert prophétique du rideau de scène en tableau, qu'il nomma "suprématiste», et révolutionna la peinture. Dans mon cerveau des phrases sont marquées au fer rouge: Les couleurs sont upleines d'exigence, elles font brûler mon cerveau» (Malevitch); uje ne cherche pas, je trouve» (Picasso); et celle-ci de Braque, moins connue: «ce qui m’a toujours sauvé, c'est que je n'ai jamais su ce que je voulais», ou bien: «ll faut que la toile efface l'idée», ou encore: «la réalité, ce n'est pas cela, c'est vers cela $\left.{ }^{2}\right)$. Autant dire que le langage a toujours été pour moi, comme pour les alchimistes autrefois, la voie d'accès à la peinture en tant que pratique, ce concept de pratique signifiant très exactement le passage à travers une matière. Quelle matière? Cennino Cennini nous en donne l'idée, au début du Livre de l'art, dans le récit de sa rencontre avec la couleur. Ce fut, au terme d'un long voyage avec son père, dans une montagne éloignée, sur le sol d'une grotte où "des veines de couleur», et notamment du blanc, "I se voyaient comme une cicatrice sur un visage ${ }^{3}$. .) Scène que jouera Louise Bourgeois, dans ses têtes en tapisserie couturées comme Le Golem, affirmant (et on la croit) qu'elle a "choisi l'art plutôt que la vie». Dans ces têtes-autoportraits, nul n'en doute, les coutures-cicatrices sont à elle, signes de l'art en elle. Les gestes y rappellent l'atelier familial de restauration de tapisseries, mais elle y rejoue sa rencontre avec la couleur, lavée dans la rivière au bout du jardin à Choisy et dans la Creuse. Connue comme sculpteur, est-elle peintre encore, dans ces têtes?

2 BAZAINE, J. Le temps de la peinture (1938-1989), Paris, Aubier, 1990, p. 71.

3

CENNINI, Cennino. Le livre de l'art ou traité de la peinture(entre I390 et I437), mis en lumière pour la lère fois par le chevallier Tambroni, trad. V. Mottez (1858), nouv. éd. augm., Paris, F. de Nobele, 1978, p. 32. 
Depuis que je ne peins plus avec une brosse ou un pinceau, que je ne recourre plus de peinture un subjectile, que je travaille des images avec l'ordinateur et réalise des vidéos numériques avant d'en avoir appris la technique (mais je sais ce que peindre veut dire, et dessiner), suis-je encore peintre? Si je ne cherche pas à peindre avec la vidéo, force est de constater que je fais à chaque fois revenir la peinture, que s'opère à mon corps défendant son transfert en vidéo, et qu'à la fin c'est elle que je trouve, sans l'avoir cherchée. Et je pense aux derniers Titien, aux derniers Goya de la Casa del Sordo, où la peinture est jetée en flaques au mépris du métier, réinventant les gestes premiers de l'art.

D'un côté donc, le langage, les mots qui nomment le monde extérieur, transfiguré par l'art en s'incarnant (en brûlant d'un feu qui ne s'arrête pas), de l'autre les «veines de couleurs» d'une grotte dans la montagne, «comme des cicatrices sur un visage» et pas ailleurs, la présence sous le uregard désarmé4 $\|^{4}$, autrement dit le monde intime devenu chair. Dans cette scène, le ublanc» donne accès à la couleur, à la chair et au sang, matières de l'art de peindre, avec tout ce qui choit du corps, et tout ce qui dans les ténèbres telluriques, au fond des océans, se métamorphose, magma visqueux qui déferle en plis et fractures gigantesques, s'engouffre et jaillit à chaque seconde. l'hypothèse est celle-ci: ma vidéo numérique Les Nageuses (6' 50, 2010; 2' 2I, 20II), qu'elle se projette ou apparaisse sur un écran plasma ou Blue Ray, pourrait être appelée peinture. Nous l'éprouverons à travers la définition de la peinture par W. Benjamin ${ }^{5}$, spécifiée par la «tache» qui ressort en devenant «marque», et les observations de Gœethe sur la couleur vivante. Comment la description de la piscine par Merleau-Ponty, modèle selon lui de la quête des peintres, s'incarne-t-elle dans cette vidéo? Comment l'intime en art, apparu selon Daniel Arasse dans le tableau de la Renaissance, a-t-il muté dans Les Nageuses? À la fin, en saurons-nous davantage sur l'acte de peindre?

LYOTARD, J. F. op. cit., p. 163.

5

BENJAMIN, Walter. Peinture et graphisme. De la peinture ou le signe et la marque (Surkamp Verlag, Frankfurt am Main, 1977), trad. P. Pénisson, La part de l'œil, dossier Le dessin, 6, 1990, pp. 13-15. Écrits français, prés. et intr. J.M. Monnoyer, Paris, Gallimard, 1991, p. 189-192.

6 ARASSE, D. Le sujet dans le tableau. Essais d'iconographie analytique (1997), Paris, Flammarion, 4è édition 2010

\section{LA MARQUE ET LA TACHE, PARADIGMES DE LA PEINTURE}

Pour Walter Benjamin la «marque» caractérise le "milieu" de la couleur dont le modèle est la rougeur de la honte sur un visage, ou les stigmates du Christ, marque-cicatrice qui possède une dimension temporelle et va à l'encontre de la personne. La couleur est aussi la «tache» qui apparaît et disparaît sur le vivant, mais aussi lorsqu'on accroche temporairement un tableau sur un mur?. Elle est ainsi le paradigme de la peinture, où «la marque est toujours absolue, et son apparition à nulle autre pareille..) En effet, la couleur se montre en tout temps "sspécifique, caractéristique, significative, sa naissance et sa détermination ne font qu'un». Ainsi la couleur relève de l'apparaître et non de l'apparence. Ce qui congédie aussitôt la question de la mimesis. ull n'y a pas une fraîcheur originaire, mais autant d'états de fraîcheur, que [1/4] de regards désarmés», précisera Lyotard. La vidéo des Nageuses, par sa rapidité, sa brièveté voulue et son montage en boucle, est à chaque seconde apparition de la couleur en train de disparaître. Ainsi la fin où la dernière nageuse étouffe dans un bain de pixels, correspond à ce que Roman Jakobson nomme la «fonction poétique», équivalence de la sélection des couleurs et de la combinaison des séquences.

Étonnant pouvoir de la couleur, qui coupe le regard en deux... Une couleur changeante, comme un regard qui se ravise, où viser «contient l'idée de voir et du visagel), du regard offensif et mortifère. Comme si notre regard tuait cette nageuse. Dans la vidéo, les deux nageuses qui se succèdent sont comme certains poissons des mers chaudes qui, juste avant leur mort, se parent de somptueux chatoiements. Entre la naissance et la mort, la couleur accompagne les transformations du vivant et la reproduction sexuée. Le sexe d'un organisme est déterminé par les chromosomes $X$ ou $Y$, dans le noyau de la cellule, particulièrement visibles dans sa phase de division.

Gœthe remarque que ula puissance des couleurs se révèle sous la forme la plus frappante en liaison avec une organisation régulière, sur les insectes dont le développement comporte une métamorphose complète, notamment les coléoptères, et de façon remarquable sur les papillons ${ }^{8} \%$. Les insectes, insectus, ont un corps sectionné, non seulement à l'état adulte, mais encore dans la structure de leur métamorphose. De la larve au lépidoptère achevé, dont

BENJAMIN, Walter, op. cit.

Goethe, op. cit., p. 205. 
les ailes portent parfois des ocelles - étrange aboutissement du processus chromatique -, la couleur est toujours là. Logée dans les entrailles, lovée dans la chysalide, elle doit rompre une carapace pour apparaître, éphémère et fragile, sur les ailes intouchables du papillon. Ainsi, pigment qui n’adhère pas à la membrane réticulée de l'aile, donc pure superficie se détachant de son support lorsqu'on y touche et que la teinte colle aux doigts, la couleur est le lieu des métamorphoses et des transmutations. Le filtre d'où s'écoule (colare) un temps réversible. Au sein de la couleur s'accomplit le chiasme entre la surface (les ailes) et la profondeur (les œufs). La réversibilité de la surface et de la profondeur s'opère aussi dans le battement des ailes du papillon, l'intermittence du cillement des ailes, où vacille la couleur. Dans cette faille où elle se fait et se défait, a lieu l'essence du regard, trait évanouissant et punctiforme, insaisissable couleur des choses, qui les peint de reflets changeants. Les choses nous regardent au gré des reflets qui les touchent, et même sur l'acier, par la grâce d'un simple souffle déposant sa buée, déferle, fugitive, une vague de couleurs. Qu'il y ait cette fugacité dans les Nageuses était mon obsession. II fallait sans relâche uréduire, réduirel, comme le voulait Duchamp, en somme, faire une vidéo «vite», qui ait la rapidité des spots télévisuels d'aujourd'hui.

Pour Benjamin, la peinture n'a pas d'arrière-fond, ce qui la spécifie est d'être une tache détachée d'un fond. Ce fond, dont elle est issue, est le déluge; le flottement de la peinture au-devant de son support procure liillusion de juste émerger du déluge. Lorsque la peinture adopte des couleurs de terre, par exemple dans les Tables de Dubuffet ou les tableaux d'Eugène Leroy, sans doute fait-elle retour à l'origine tellurique des couleurs, à cette dimensionnalité où a lieu la déflagration de l'être retournant au gouffre que l'artiste, ressent plus que tout autre. Profondeur dont le plongeur doit revenir, dit Valéry. Profondeur que Dubuffet constelle avec des ailes de papillons. Puisque l'artiste n'est pas un papillon aux éclatantes couleurs, où doit-il aller chercher sa couleur, depuis toujours? Lacan nous le souffle?: dans le déchet, la viscosité des matières désintégrées, broyées, dissoutes et oxydées, digérées, putréfiées dans l'eau, cuites par la chaleur, le feu ou le soleil. Dans la boue et les viscères. C'est là où dès les commencements, la couleur se fait. Ce sont la couleur native de Duchamp, couleur du chocolat que le célibataire broie

\section{9}

LACAN, J. Le séminaire. Livre XI. Les quatre concepts fondamentaux de la psychanalyse (1964), paris, Seuil, 1973, p. 107.

10

Dictionnaire Robert. lui-même... et les couleurs-matières des Nageuses.

Parce qu'elle ressort, et spécialement dans la peinture, la couleur fait oublier le sans- fond primordial d'où elle vient: l'abîme, par définition sans fond. Le rouge au début des Nageuses y est semblable au dernier coup de pinceau pour Frenhofer: au peintre, on ne sait aucun gré des dessous, qui affleurent en surface, comme dans la gorge des noyés... Si, comme le disait Roger de Piles, la couleur est un fard, ce ne peut être selon l'expression: «piquer un fard», elle est le pharmakon dont on maquille les cadavres, donnant l'illusion de la vie. Elle doit pourtant donner l'impression d'avoir "germé" sur le support, d'être une zôgraphia, une peinture du vivant. Klee multiplie dans ses tableaux les couches de fonds, et les couleurs de Poussin semblent émerger des ténèbres pour y retourner. La naissance de la vie à même la mort, c'est peut-être aussi le scandale d'Olympia: la couleur cadavérique du corps de Victorine Meurent, qui dit la possible putréfaction de la chair, l'origine dégoûtante des couleurs, la mort sur le vivant, et qui, décapitée par le ruban autour du cou, nous regarde droit dans les yeux. Les couleurs des Nageuses, émergeant des ténèbres, nous regardent de leurs fréfonds. Cette couleur -matière, les anciens le savaient, qui la mettaient sur l'architecture, dans un geste archaïque défiant la mesure et les calculs savants, était l'inassimilable, l'irruption du tellurique au fronton des temples, de l'animalité dans l'œuvre de l'esprit. Rupture qui nous met face à l'être sauvage, ce tissu commun dont nous sommes faits, qui se tient devant nous dans le monde vertical. Pour Benjamin, la peinture est verticale, elle est ce qui du monde se lève et regarde la lumière. Elle veut oublier son origine tellurique, que le dessin, demeuré horizontal, plus près du gouffre, lui rappelle. La piscine est-elle ce gouffre?

\section{FILMER LA PISCINE. RAYONNEMENT DU VISIBLE}

À la volée, depuis la fenêtre de ma chambre d' hôtel à Manama, capitale du Bahrein, où je suis, étrangère de passage, pour une exposition personnelle en mars 2008, je filme en plongée deux nageuses dans la piscine. On sait qu'une œuvre doit retracer la totalité de l'histoire du sujet qu'elle traite (ici la piscine et deux nageuses). Bassin pour les rites purificatoires» chrétiens, dont le poisson (piscis) est l'emblème, la piscine est dans la liturgie, «une petite cuve destinée à recevoir l'eau qui a servi aux baptêmes, à la purification des objets sacrés ${ }^{10}$. .) Grand bassin de natation depuis le

II

MARLEAU-PONTY, M. L'œil et l'esprit, Paris, Gallimard, 1973, p. 70-7I. 
XVlè siècle, devenue aujourd'hui signe d'élévation sociale, la piscine est aussi le bassin de désactivation d'un réacteur dans une centrale nucléaire. Cette constellation des sens du mot piscine, ses mutations du religieux au social et au scientifique, du nucléaire où la piscine désactive et purifie l'énergie atomique de son possible danger de mort, franchit d'un mot l'histoire des substances et installe une connivence entre le sacré, l'atome et le pixel. Selon Merleau-Ponty, «l'eau elle-même, la puissance aqueuse, l'élément sirupeux et miroitant, je ne peux pas dire qu'elle soit dans l'espace: elle n'est pas ailleurs, mais elle n'est pas dans la piscine. Elle l'habite, elle s'y matérialise, elle n'y est pas contenue, et si je lève les yeux vers l'écran des cyprès où joue le réseau des reflets, je ne puis contester que l'eau le visite aussi, ou du moins y envoie son essence active et vivante. C'est cette animation interne, ce rayonnement du visible que le peintre cherche sous les noms de profondeur, d'espace, de couleur".. Cette "animation interne», ce urayonnement du visible» retentissement, dans l'esprit, de tous les sens du mot piscine. Ainsi dans Villa Amalia (2009) l'héroïne, Éliane, compositrice de musique, étouffe dans sa propre vie. Elle porte dans une piscine urbaine des lunettes de plongée qui la font ressembler à un poisson forcé de sortir de l'eau pour respirer. Elle trouve enfin sa respiration créatrice dans une villa sur une île, face à la Méditerranée. La mer enfin trouvée baigne la totalité de l'écran de cinéma, qui devient cette eau salvatrice, purificatrice, mais cadrée, désactivant la menace d'étouffement.

J'ai filmé à leur insu deux nageuses, l'une en maillot noir jusqu'aux genoux (qui en sortant revêtira la abaya), l'autre en deux-pièces à fleurs. L'eau devait être tout entière contenue dans l'écran de contrôle, qui a remplacé la fenêtre du tableau d'Alberti, les nageuses demeurant dans cette eau écranique, virtuelle, modèle réduit de toute l'eau dans laquelle on puisse se baigner. Comme si je filmais deux spécimens de nageuses immergées dans un liquide de laboratoire dont je modifierai plus tard la substance par la couleur, à l'aide de l'ordinateur, selon le protocole d'une rigoureuse expérimentation, qui donnerait toute sa chance à la surface en faisant affleurer sa profondeur dans ses reflets. Le point de vue éloigné fait reculer la scène dans le temps. Dès que je les filme, les deux nageuses sont sur le disque dur de la caméra et dans le passé qui recule jusquà la faille qui forma le Golfe Persique où s'engouffrèrent les eaux salées. L'appareil transfigure la scène en système binaire $(0, I)$. II

12

Paolo Ucello, Les scènes de la vie de Noé: Le Déluge et le Retrait des eaux, XV siècle, $206 \times 170 \mathrm{~cm}$, Chiostro Verde, Santa Maria Novella, Florence. capture l'indifférenciation mathématique de l'eau et des nageuses, que je vois prisonniëres de l'écran, tournant sans fin dans cet espace confiné. L'eau n'est pas dans la piscine, ni sur l'écran, mais dans l'intimité cosmique de l'univers auquel le numérique donne accès. Quand ma caméra suit les nageuses afin qu'elles ne sortent pas de l'eau écranique, je pense aux meurtres dans les piscines au cinéma: Les Diaboliques (1955), La Piscine (1968), la série des James Bond (1962-2008), aux noyés du Titanic (1997), et surtout à ceux qui tentent de nager et se sauver dans la fresque du Déluge du cloître de l'église Santa Maria Novella à Florence ${ }^{12}$. Fresque abîmée dans ce cloître ouvert sur un petit jardin, que je suis allée voir, gorge nouée: pour moi le vrai déluge est là, c'est en peinture qu'il existe, dans cette fresque pâlie en partie effacée ouverte à tous les vents depuis près de cinq siècles. Dans le lignage de ces films, de cette fresque, la caméra capture la jointure du milieu et du vivant où se joue le drame de l'existence. Chez l'être humain, remarque Dagognet, philosophe et médecin, ule dedans de l'ex-dehors permet le dehors de l'ancien dedans ${ }^{13} .$. En effet l'être humain n'est plus protégé par les os ou leurs équivalents protecteurs qui enveloppaient le corps à l'origine du vivant. Je suis résolument du côté de cette fragilité. C'est elle que je veux magnifier, comme dans la fresque du Déluge, pour qu'émerge une précarité partagée depuis cette époque. II faudra saisir à l'état naissant, ainsi que s'est formée l'île où je suis, le décalage entre la réalité concrète et sa puissance de transfiguration. Ce sera la tâche des calculs de l'ordinateur, des couches de couleur, qui altèreront profondément la définition des images, les faisant reculer à de lointaines époques où on ne comptait pas les pixels par millions. Les faisant, simultanément, devenir peinture: milieu chromosomique en miroir de nos corps, tache en mouvement, marque qui ressort sur du vivant.

La capture numérique, si bien nommée, exile les nageuses des yeux de l'esprit comme de ceux du corps, uen soumettant leur apparence à la force inhérente à la distance ${ }^{14}$. I) En tant que données mathématiques, elles sont traduites en schémas identiques aux structures mentales humaines. Les nageuses et l'eau mutent en symboles issus de lois cosmiques universelles; elles ont leur point de référence hors de la planète, échappant à la géométrie, mesure de la terre. Leur point

13 DAGOGNET, F. Philosophie d'un retournement, Fougères, Encre marine, 200I, p. 33.

14 ARENDT, H.. Condition de l'homme moderne (196I), trad. G. Fradier, préf. P. Ricœur, Paris, Calmann-Lévy, 1994, p. 335-338. 
de vue n'est pas le mien, en contre-plongée, géographique et privé (point de vue d'étrangère), mais celui de l'astrophysique universelle. Ce point hors-champ, je le vois dans l'image filmée, quand chaque nageuse soulève au plus haut la tête hors de l'eau comme pour éviter de se noyer. Ce moment est pour moi le punctum, ce qui me touche. Leur prison est l'eau, est la terre, c'est la mienne, c'est la vôtre. Mon premier souvenir, enfant, est la terre où sont posés mes pieds, au loin la mer à traverser pour aller au ciel, là-haut. Moi si petite. La caméra numérique m’apporte enfin le point de vue du ciel, fait éprouver la même loi de gravitation de Newton pour les corps terrestres et célestes. Ce n'est pas le ciel de mon enfance, mais un ciel simulé. Mathématique. La peinture est devenue cosmique.

\section{MUTATIONS DE L'INTIME EN PEINTURE}

L'intime a été conçu et pensé dans la peinture au XVè siècle, à la Renaissance, avec l'instauration du tableau moderne, défini par Alberti comme ufenêtre ouverte» sur le monde. Donnant à cela la plus grand extension, G. Wajcman uenvisage que le tableau moderne aura, d'un même geste, instauré l'idée cartésienne que l'homme a désormais droit de regard sur le monde, avec Dieu, et défini l'intime comme ce lieu dans le monde où l'homme peut se tenir séparé du monde, d'où, par la fenêtre, en secret, il peut le contempler, et où, hors de tout regard, il peut se regarder lui-même $e^{15}$.I) A cette époque la peinture devient un art libéral par l'introduction des mathématiques, grâce à l'usage de la perspective. Par la fenêtre albertienne qui permet une vue, le monde est représenté selon les lois de la perspective «légitime», issue de la géométrie, par définition mesure de la terre. Afin d'explorer l'invention de l'intime dans le tableau de la Renaissance, Daniel Arasse rappelle cette idée de Brunelleschi: «Tout peintre se peint ${ }^{16} . »$ Savonarole précise: «en tant que peintre», «selon son concept.») Formule en vogue à Florence autour de 1500. Comment l'intime de la peinture a-t-il muté avec l'usage de l'ordinateur, dans un monde non plus représenté mais simulé par le numérique? Où l'écran de contrôle de la caméra a remplacé la fenêtre d'Alberti? Selon quel procès la vidéo numérique, Les Nageuses ${ }^{17}$ (2010-20II), rend-elle à leur puissance contemporaine, infiltrée par la science, les couleurs de la chair et du sang, des

15

http://www.lacan.com/symptom8_articles/wajcman8.html. Consulté le 19-10-2011.

16

ARASSE, D. Le sujet dans le tableau, Paris, Flammarion, 2010, p. II. ténèbres telluriques et du feu magmatique?

Blanchot récusait «l'intimité fermée», ce "mauvais intérieur» qui nous exclut de l'accès véritable aux choses. II demande d'atteindre ce point que Barthes nommera le punctum et désignera comme blessure intime, charnelle, qui est dans l'œurre et cependant horschamp. La couleur en est la cicatrice, elle se peint sur le visage des regardeurs, grâce aux neurones miroirs, découverts en 1996 par le neurologue Rizzolatti. Dans l'intervalle de ce transfert, a lieu ce point d'origine de l'œuvre: «à la fois intimité et dehors; une intimité qui, en nous, serait la réalité du dehors, telle que nous y serions en nous au-dehors dans l'intimité et l'ampleur intime de ce dehors ${ }^{18}$. .) Dans la vidéo, je veux qu'elles nagent à l'intérieur de leur propre corps et à l'extérieur d'elles-mêmes, dans cette intime réversibilité du vivant (Dagognet). Elles s'y déplacent comme dans l'univers où l'on ne rencontre que soi-même, où l'on ne voit rien d'autre que ce qui se ramène à des schémas que l'être humain porte en lui. À l'inverse de la science, il n'y a pas de progrès en art; aux symboles mathématiques se superposent les mythes, autres constructions de l'esprit. Les Nageuses se déplacent tout d'abord dans un bain rouge vif, dans leur propre sang, donnant l'impression de faire naître, de leurs mouvements, la couleur, transfusant le rouge vivant à la sortie de sa prison de veines et de chair. Le processus générique est aussi la permanence, restée plus vive chez l'artiste, d'une mémoire fætale. Dans un mythe dogon, on dit que durant les derniers mois, le fætus nage dans le ventre de sa mère ${ }^{19}$. Les Nageuses sont-elles en train de se donner la vie, dans leur propre sang qu'on dit impur pour les femmes, comme l'artiste qui naît de son œuvre? Au cours du travail, la vidéo inverse la fonction de purification des piscines dans les centrales nucléaires, où s'effectue la désactivation des impuretés, d'où le son, métallique et sourd, pourrait provenir. La lenteur et le son transmutent les Nageuses en corps-machines, les immergent dans d'épais liquides: sang, nappes de pétrole, abysses pourpres, lave en fusion qui consume la nageuse, dans l'avant-dernière séquence. Alors Les Nageuses entrent dans l'intimité d'une «mort partagée, archétypale, qui résonne dans l'espèce entière ${ }^{20} .11$

\section{7}

Les Nageuses, vidéos numériques, 6'50", 2010 et 2'21, 2011 .

\section{8} BLANCHOT, M. L'Espace littéraire, Paris, Gallimard, coll. Folio Essais, 1955, p. 173.

19 DURAND, G. Les Structures anthropologiques de l'imaginaire, Paris, Dunod, 1969, p. 247. 
Sang, pétrole, lave, reconnaissables à leurs couleurs, ne sont pas représentés mais simulés. Du traitement numérique advient une sombre histologie du corps, fusion du code digital et du code génétique, issue des grandes fosses abyssales où naquit la vie. Par les pixels visibles de près dans les dernières secondes (Nageuses 20II), Les Nageuses sont fabriquées, dérivées, comme Pandora. Ainsi les riches étoffes numériques dont celle-ci aurait pu être vêtue simulent le retournement de nos tissus et liquides physiologiques dans le chatoiement de la substance profonde de la nuit cosmique. Exposées dans un espace d'art du marais à Paris ${ }^{21}$, Les Nageuses font apparaître, plus intensément dans la nuit dissoute que sont les couleurs dans le régime nocturne des images, entrelacées avec la rue, la ville, son histoire sanglante sous l'Ancien régime et la révolution, le fleuve qui a charrié des corps d'algériens le 17 octobre 1961 - je m'en souviens -, ce que Merleau-Ponty nomme ula chair du monde.» Nocturne, océanique, cosmique, antédiluvienne, elle scintille aux origines de la vie confondue avec l'art.

La vidéo numérique accomplirait la scintillation de notre altérité dédifférenciée, où la poiétique retrouve le chemin du sang d'une lignée artistique, d'une généalogie mythique et d'une chair cosmique. En s'incarnant dans d'autres corps, l'artiste descend dans le paysage liquide comme le sang dans les veines, incorpore ce paysage virtuel où son corps ne trouve pas sa place, empourpre ule ciel qui fuit / $\dot{A}$ travers un carré de glace ${ }^{22} \|$ qu'a regardé à Reading, "lavec passion», le condamné à mort célébré par Oscar Wilde dans la Ballade de la geôle de Reading. Mais dans Les Nageuses, à notre époque où pour la première fois ce pourpre est simulé par des calculs, le ciel est devenu scientifique. Ce qui n'annule pas les pouvoirs prémonitoires de l'art. Exposées pour la première fois en 2010, peu avant l'éruption de l'Eyjafjoll en Islande, la fuite du pétrole dans le Golfe du Mexique, les incendies de Russie et les inondations du Pakistan, annonçant la catastrophe de Fukushima, Les Nageuses, reprises en 20II, en deviennent la prophétie, donnant à voir hors-champ les

20

Ibid., p. 253.

21

Le Dressing, Espace d'art Free'P'Star, ler-3I mai 2010, nuit et jour.

22

WILDE, Oscar. De profondis. La Ballade de la geôle de Reading (Librairie générale française, 2000, 1905 et 1962 pour De profondis; 1898 pour La Ballade), précédés de L'Artiste en prison d'A. Camus, trad. et préf. L. Lack et J. Besson, Paris, Le Livre de Poche, coll. Classique, 2004, p. 213. ténèbres contemporaines, sous l'aspect de leur menace que nos corps abritent. Elles transfusent entre eux, à la vitesse de la lumière de leurs pixels, non pas les souvenirs privés de l'enfance, ni les scènes publiques de l'histoire, mais ce que Rilke nomme «l'intimité du cœurn: intimité d'une anamnèse magmatique, de la nuit cosmique et de la profondeur océanique commune à tous les arts. Cette profondeur n'est pas dans la piscine, mais dans les couleurs de la vidéo et leur transsubstantiation en nous. Dans nos chromosomes et notre ADN qui nous est mondialement commun à $99 \%$.

Seul l'art, qui procure à la chair et au sang l'indifférenciation des genres et des époques, s'élève comme le voulait Mallarmé à la puissance du ciel étoilé qui contient, virtuellement, la multiplicité des galaxies de plus en plus anciennes que les télescopes de plus en plus géants permettent d'approcher, à l'exception de la «matière noires indétectable par l'astrophysique. Composant $95 \%$ de l'univers, elle peut seulement être simulée numériquement. Avec le numérique, qui en simule les couleurs, même la chair et le sang s'extraient des lois cosmiques excédant le système solaire. Distinctes des lois planétaires et naturelles, elles transfigurent durablement douleur et précarité en les donnant à voir dans le rythme vivant du coloris. Numérique ou pas, l'art œuvrerait avec cette matière noire, ou masse manquante de l'univers, riche de toutes les teintes celées dans l'obscur, incalculable. Cette matière noire, comme les noirs de Malevitch ou de Matisse, est la couleur du caché de la chair. C'est elle qui permet l'incarnation démontrée par les neurosciences: dans notre cerveau, des neurones miroirs provoquent en nous, à travers notre regard, l'esquisse du geste - ici des nageuses faisant surgir en boucle, du noir, ses couleurs, pendant 2 minutes 21 secondes. Alors, si la vidéo peut être projetée au revers d'un très grand écran, avec un son de machine résonnant comme dans un formidable chantier tellurique, sorte d'enfer, l'on doit éprouver la sensation de se tenir au milieu de la couleur en train de se faire dans un bruit assourdissant. Dans la couleur comme milieu, en somme, détachée d'un fond, ainsi que Benjamin l'a définie.

Que donne à voir, comme tout art, cette vidéo-peinture? La disparition des êtres et des choses dans l'intimité de leur mort, leur incarnation virtuelle et réelle à la fois. C'est pourquoi, comme la matière noire que l'on nomme aussi masse cachée, quand l'œurre se montre en public, cette intimité doit rester masquée. En latence derrière le sensible.Virtuelle. Inapprochable. Sacrée. Elle reste cette uintimité du cœurn, au sens où le cœur est aussi un organe, et où cet organe est ce qui "pense ${ }^{23}$ ") en premier, activé par une région particulière du cerveau. L'intime en art n'existe qu'incarné, ne s'incarne que dans le regard d'un public. L'art n'a rien à faire avec les souvenirs de 
la vie privée ni avec les circonstances de la vie sociale, seulement avec la vie intime de l'art, de geste en geste, avec des moyens renouvelés par chaque époque, sans que les moyens traditionnels en deviennent obsolètes, puisqu'il n'y a pas de progrès en art. II faut cependant, de quelque façon et sans relâche, revenir aux origines, à ce qu'on nomme, dans les mythes, «avant le déluge», que provoque l'artiste et qui s'y plonge. Victime et bourreau.

«Dans la vie réelle [dit Louise Bourgeois], je m’identifie à la victime, c'est pour ça que je me suis tournée vers l'art. Dans mon art, je suis l'assassin. Je sympathise avec le sort du meurtrier ${ }^{24}$. " Les Nageuses, c'est avec des couleurs picturales que je les assassine, calcinant l'une d'elles dans la lave brûlante. La piscine écranique est-elle le «tableau parfait», comme on le dit de celles de David Hockney? Dans Portrait d'un artiste (Piscine avec deux figures) de $1972^{25}$ ule personnage dans la piscine devient une allégorie de la figure peinte, et la piscine, le symbole de l'art ${ }^{26} . \lambda$ Certes, mais nous pouvons voir dans ce tableau de Hockney une scène de meurtre, où le nageur est un noyé dont l'assassin debout au bord de la piscine, en veste rouge, vérifie du regard qu'il ne bouge plus. L'artiste se serait dédoublé en deux figures. II faut, dit-il, use ressentir soi-même et peindre: sortir de soi en quelque sorte et se placer dans la peinture. [1/4] j’ai toujours été obsédé par le fait de voir [1/4] L'art visuel doit traiter du fait de voir parce que la première chose que vous demande l'art visuel est de regarder ${ }^{27} . .1$ Regarder le crime parfait? À la fin du XVè siècle, pour Marcile Ficin, fils de médecin, ules œurres d'art qui se rapportent à la vue et à l'ouïe proclament l'esprit de l'artiste», c'est-à-dire "la disposition» et «l'image de son esprit», car «l'esprit s'exprime et se reflète

\section{3}

CASSIRER, E. Langage et mythe. $\dot{A}$ propos des noms de dieux (1924), trad. 0. Hanse-Love, Paris, Minuit, 1973, p. 63-64.

\section{4}

Louise Bourgeois: sculptures, environnements, dessins 1938-1995, Paris, Musée d'art moderne de la ville de Paris, 1995, p. 49.

\section{5}

David Hockney, Portrait d'un artiste (Piscine avec deux figures),I972, acrilyque sur tole, $214 \times 305 \mathrm{~cm}$, coll. particulière.

\section{6}

David Hockney. Espace-paysage, catalogue d'exposition, Paris, centre Georges Pompidou, 1999, p. 96. comme dans un miroir.» Miroir dont il n'est pas étonnant que la piscine soit le paradigme dont Merleau-Ponty fournit la description: "Quand je vois à travers l'épaisseur de l'eau le carrelage au fond de la piscine, je ne le vois pas malgré l'eau, les reflets, je le vois justement à travers eux, par eux. [1/4] Si je voyais sans cette chair la géométrie du carrelage, c'est alors que je cesserais de le voir comme il est, où il est, à savoir: plus loin que tout lieu identique ${ }^{28} . \nu$ Hockney dit encore à propos d'une autre de ses piscines: Gregory nageant. Los Angeles, I I mars $1982^{29}$, qu'il l'imagine «comme étant [sa] version des plafonds de Tiepolo [1/4] vous savez, ces voûtes baroques recouvertes de petits anges dodus qui montent très haut dans le ciel si bleu ${ }^{30} . \lambda$

\section{INTIME MUTABILITÉ DE LA MATIÈRE PICTURALE}

Dans L'inhumain, Lyotard, qui savait regarder la peinture, écrivait: "Le peintre est saisi ou dessaisi par une teinte ${ }^{31} »$. II prenait pour exemple Cézanne, qui devant la montagne [1/4], voulaitque ce dessaisissement, le regardeur puisse à son tour l'éprouver [1/4] devant la couleur posée et composée en tableau.» Et quand je dis couleur [ajoutait Lyotard], cela s'entend de toute matière picturale.I À l'inverse de Cézanne devant la montagne, c'est au cinéma et le plus souvent par la télévision, qu'éruptions volcaniques et tsunamis, catastrophes en tous genres, ont pénétré, en images provoquées par les neurones-miroirs, au plus intime de «ce qui se voit en moi» (Merleau-Ponty). J'ai voulu redonner à ces images-miroirs, immatérielles et lumineuses, bien réelles pour moi, leur intime

27

Ibid., p. 196.

28 MARLEAU-PONTY, M. L'œil et l'esprit, Paris, Gallimard, 1973, p. 70-7I.

29 Gregory nageant. Los Angeles, I mars 1982, polaroïd composite, 70,5 × 130,2 cm, coll. D. Hockney

30

Hockney, op. cit., p. 197.

31 LYOTARD, op. cit., p. 163. 
matérialité picturale, chromosomique, à travers la mutabilité de la couleur "qui la rend propice au désarmement du regard ${ }^{32}$ », à la «défaite de l'esprit». Conditions d'un devenir-muqueuses, organes, battements de cœur, viscères. Ce avec quoi en définitive on peint. Les mutations d'une piscine et des nageuses ne sont pas un prétexte pour expérimenter l'intime mutabilité de la matière numérique.

Est-ce un hasard si j’ai filmé en 2008 au Bahreinn, ce pays du Golfe secoué trois ans plus tard, comme d'autres, par le «printemps arabell? Si j'ai vu, dans deux nageuses dont les différences d'origines m'ont frappée, la violence du conflit à venir? Conflit sur lequel une chape de plomb est tombée. Ce que j’ai vu, ce n'est pas le bleu de la piscine d'un hôtel de luxe pour touristes fortunés, mais sa part d'ombre, sa tragédie latente. Comme un appel. De même Hockney, qui fut graphiste ${ }^{33}$, qui a longtemps expérimenté la couleur avec la photocopieuse et l'ordinateur, a fait virer le bleu des piscines californiennes et du ciel des plafonds baroques en ce rouge sanglant dont sont peintes ses immenses toiles du Grand Canyon ${ }^{34}$, plaies à vif des origines à ciel ouvert. Sous la très mince bande de ciel bleu qui court sur les 744,2 cm de long du Bigger Grand Canyon, nous voyons sa chair vomie par les bouches ouvertes d'un couple de damnés, dans un double cri érectile.

La condition pour être contemporain? Ne pas use laisser aveugler par les lumières du siècle et [parvenir] à saisir en elles la part de l'ombre, leur sombre intimité35. In S'en saisir en peinture, par contact intime avec la matière picturale, fut-elle produite par un code, sans craindre le risque de s'y perdre, puisqu'il faut précisément disparaître dans la peinture comme le peintre chinois dans le paysage. Devenir comme la nageuse qui se noie dans les pixels, bouche ouverte, et renaît dans son sang. "Entre le monde et moi, choisir le monde», disait Kafka. Faire émerger un monde en peinture, même avec les moyens de la vidéo numérique. Hockney, impressionné par une

32

lbid.

33

II obtint à Bradford le National Diploma of Design, a fait des décors d'opéra et des affiches.

34

A Bigger Grand Canyon, 1998, huile sur 60 toiles, 207 x 744 cm, coll. D. Hockney.

35

AGAMBEN, G. Qu'est ce que le contemporain?, trad. M. Rovere, Paris, Payot et Rivages, coll. Poche, 2008, p. 21. exposition de Picasso à la Tate Gallery, dira: «ll pouvait maîtriser tous les styles, toutes les techniques. La leçon que j'en tire, c'est que l'on doit les utiliser tous ${ }^{36} . \|$ Façons de revenir, à chaque fois, aux origines génériques de l'art, à un champ élargi de la peinture, véritable corps de l'artiste. Façon, comme Louise Bourgeois, de «choisir l'art plutôt que la vie».

26 octobre 2011

36

D. Hockney, op. cit, p. 169.

「了

ÉLIANE CHIRON: est agrégée d'Arts plastiques, Docteur d'État, Professeur émérite à l'université Paris I Panthéon-Sorbonne et artiste plasticienne. À Paris I, elle dirige le Centre de Recherche en Arts Visuels (CRAV). Sa recherche, croisée avec une pratique artistique, questionne le procès créateur et les mutations du regard, notamment à l'ère du numérique. Elle publie et expose à l'international (Brésil, Chine, Tunisie, Corée, etc.). Elle a dirigé aux Publications de la Sorbonne une série d'ouvrages sur $X$, l'œuvre en procès: Croisements dans l'art (1996), Croisements des arts (1997), L'incertain dans l'art (1998), La main en procés dans les arts plastiques (2000), L'objet et son lieu (2004). Paysages croisés. La part du corps (2008), Migrations / Mutations. Paysages dans l'art contemporain (2010), L'intime, le privé, le public dans l'art contemporain (2012). À paraître, un ouvrage personnel: l'énigme du visible. 